\title{
Evaluating the Utility Core in the Prefabricated Housing Industry - past, present and future
}

\author{
Carlo Carbone \\ Basem Eid Mohamed
}

\begin{abstract}
This paper proposes an extensive review of literature, inventions and exploration in order to detect new potentials for designing integrated, technology-driven, flexible and adaptable prefabricated utility cores for today's housing industry. Harnessing, distributing, tempering and supplying water, heat and power in a building produces its share of design, technical and coordination issues. The relationship between services and architectural space has long challenged designers and manufacturers. Throughout construction history, and modern architecture in particular, the wet service core or utility core was a recurring instrument for efficiently zoning services; the utility core epitomized rationalization within a self-contained engine-like device positioned to serve the entire dwelling. The core was intended as a hub accommodating mechanical and technological equipment, electrical services, plumbing fixtures, water supply, drain, waste, vent piping, telephone cables, and easy connections to site infrastructure. Today's industrial capabilities and building information modeling allows the core to be redefined in relation to multiple scales and various organizational possibilities with regard to space/function connections. Further an adaptable core articulated to the open building theoretical framework of layering systems to avoid entanglement and to maximize durability can be part of a comprehensive strategy to enable customization. This review of literature and precedents contributes to a robust historic
\end{abstract}

\section{Permissions and copyright}

Authors retain copyright and grant the journal right of first publication with the work simultaneously licensed under a Creative Commons Attribution License that allows others to share the work with an acknowledgement of the work's authorship and initial publication in this journal (Attribution-ShareAlike).

Creative Commons Attribution 3.0 Unported (CC BY 3.0)

You are free to: Share - copy and redistribute the material in any medium or format. Adapt — remix, transform, and build upon the material for any purpose, even commercially. The licensor cannot revoke these freedoms as long as you follow the license terms.

Under the following terms: Attribution - You must give appropriate credit, provide a link to the license, and indicate if changes were made. You may do so in any reasonable manner, but not in any way that suggests the licensor endorses you or your use.

No additional restrictions - You may not apply legal terms or technological measures that legally restrict others from doing anything the license permits.

How to cite:

Carbone, Carlo and Basem Eid Mohamed. 2017. "Evaluating the Utility Core in the Prefabricated Housing Industry - past, present and future." Enquiry 14 (1): 37-47. narrative of two distinct approaches of architectural rhetoric and industrial production. This paper will endeavor to illustrate this narrative and evaluate the potentials for achieving broader application.

Keywords: Architecture, Housing, Prefabrication, Utility core, Building Technology, Manufactured Building, Industrialized Building, Building Information Modeling, Mass customization.

\section{INTRODUCTION}

The post-World War II housing boom experienced by a majority of industrialized countries leveraged military advances toward a new consumerism and democratized luxuries such as indoor plumbing, central heating and electrical distribution. The house was no longer simply for occupant protection; the industrialized house's technologically advanced user-friendly devices allowed for cooking, heating, lighting, leisure and even telecommunication (Topping, Lawrence, Spencer, Larson, and McLeish 2004, 6). Moreover, various services developed as 20th century technology progressed. Each new layer of technology appended building processes obscuring the relationship with other services creating an ever-increasing entanglement: services are intersected with little planning, creating difficult coordination conditions and impeding future retrofit options (Kendall and Teicher, 2000, 33). The freedom and ease of use associated with modern services and amenities complicated traditional building techniques and the added components presented challenges for conventional building trades. The distribution of wiring, ducts, and equipment brought with it the fairly recent idea of building service coordination and trade organization specifically. The network of services often left to on-site building created a muddled relationship between structure, envelope and mechanical elements, which still persists (Topping, Lawrence, Spencer, Larson, and McLeish 2004, 50). Furthermore the ever-increasing technological nature of our dwellings accompanied with the evolving nature of contemporary lifestyle accelerates the need for retrofitting, which is further complicated by service entanglement and argues for a systematic strategy for service integration.

In the pursuit of adequate solutions, architects, builders and manufacturers idealized the prefabricated service core as a strategy 
for simplifying infrastructure provision. Richard Buckminster Fuller's Integrated Bathroom Unit exposed in 1916 a simple core strategy packaging services into a fragment able to supply any building type. Both architecture and industry spread this idea into countless variants. This essay examines the generative correlations between architecture, industry and the efficient organization of dwellings' service spaces. Abundantly documented but still marginally applied, the utility core can be an important device in rationalizing service distribution and activating dwelling flexibility and adaptability through open planning. This paper aims to contribute to elevating the core's practical and theoretical importance and to preview some current drivers for development.

\section{OBJECTIVES}

Offsite construction in its many forms is often cited as a major part of building sustainably. Prefabrication through DFMA (Design for Manufacturing and Assembly), modern methods of construction, lean construction and BIM are all driving a new era of attempts to revolutionize building culture (Kieran and Timberlake 2004) ${ }^{1}$. In the context of current productiveness, it seems of particular interest to make sure the industry considers its past attempts in order to use what has been done to inform a more successful present and future. Discussing prefabricated architecture's past failures, Kieran and Timberlake's Refabricating Architecture (2004) illustrates the importance of research in examining past efforts in insuring future innovation. This paper examines the particular segment of offsite construction related to the production of rational units containing buildings' services in order to achieve a greater efficiency in matters of design and construction. Identified under many headings, including core, service core, modular mechanical unit, and heart units, among others, the prefabricated utility module has endured over the past century as an object of study. Furthermore, as the housing industry slowly incorporates contemporary design and fabrication tools, it is possible to envision an uncomplicated mass-customizable package smartly positioned and potentially data informed as a design device to optimize service coordination and spatial planning. As the industry evolves, however, little attention is accorded to more than a century of exploration. Studying and framing the inventiveness of certain past systems will complement the existing literature and help identify areas of potential innovation.

The robust historical narrative that emerges from our literature review reveals the various designations - packaged power plant, the home's engine, the mechanical wing ${ }^{2}$ - elucidating the ideal of rationalizing services into a simple architectural device. An initial analysis of prototypical projects, scholarly work, and construction strategies made a compelling argument for a literature review which is divided into three

\footnotetext{
${ }^{1}$ See also SmartMarket Report, Prefabrication and Modularization: Increasing Productivity in the Construction Industry, McGraw-Hill Construction retrieved on June 25, 2017 from - http://www.modular.org/htmlPage. aspx?name=McGrawHill_Prefabrication .

${ }^{2}$ The mechanical wing is a theoretical project by R. Buckminster Fuller which proposed a mobile utility core. It was first published in Architectural Forum; October 1940.
}

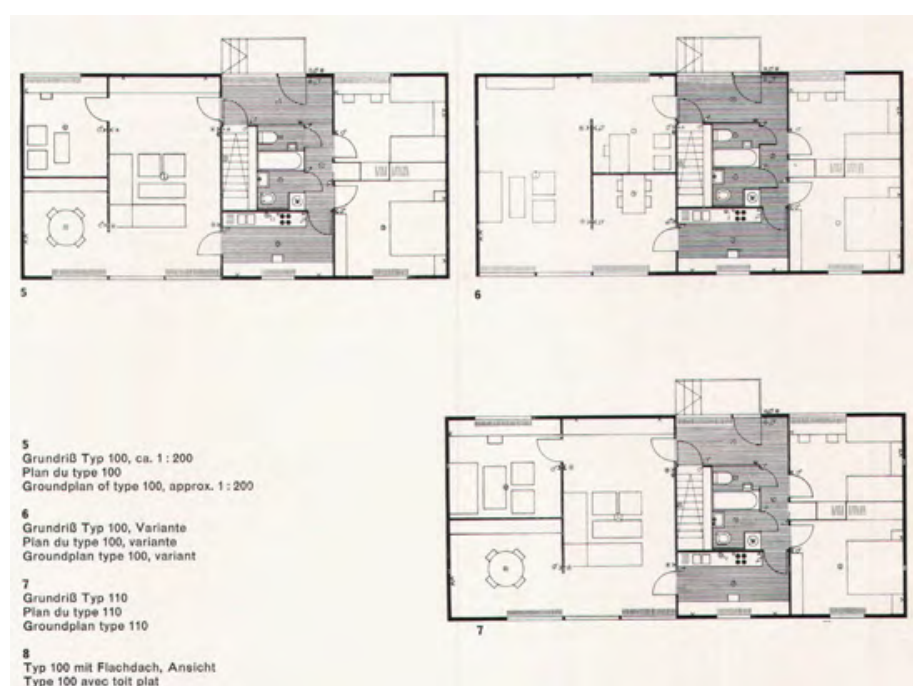

Figure 1. Quelle Bungalow Plan. Source: (Ebert 2008)

parts: the first part contains a review of certain fundamental principles which have highlighted a century of discourse; the second part comprises a comparative assessment of architectural or manufacturing experiments in order to illustrate the beginnings of a catalogue of spatial strategies; and the third part examines patented inventions relating to the core. Although some of the studied prototypes have also been patented, a study of thirty-seven patents argued for their inclusion as an important component of our review. The patents reveal the core's exploration as a specifically technological device to process building services. A critical review of these ideas will help determine areas of potential action in order to actualize the utility core and imagine its relevance as a manufactured component in designing personalized dwelling spaces.

Our analysis relates a narrative of three strategies: architecture, manufacturing, and on-site building, which tend to operate independently. The different fields rarely seem to evolve symbiotically, however today's BIM (Building Information Modeling) technology seems to create this perfect storm, as was the case at the beginning of the twentieth century, another era of architectural and industrial convergence. Beyond the analysis and literature review, the paper postulates current technology as a future space for exploration.

Recently, efforts in incorporating high-tech utilities and mechanical systems in buildings, specifically in the residential sector, have augmented challenges in coordination and integration. Growing demand for individualization, coupled with changing lifestyles and multiple family evolution patterns argues for variable and flexible design practices. The utility core in this setting could be an informed part of design processes, creating variable housing patterns based on mechanical hubs. It is evident that such an approach is lacking in the prefabricated housing industry, due to difficulties in synchronizing design variants with technical and production requirements 


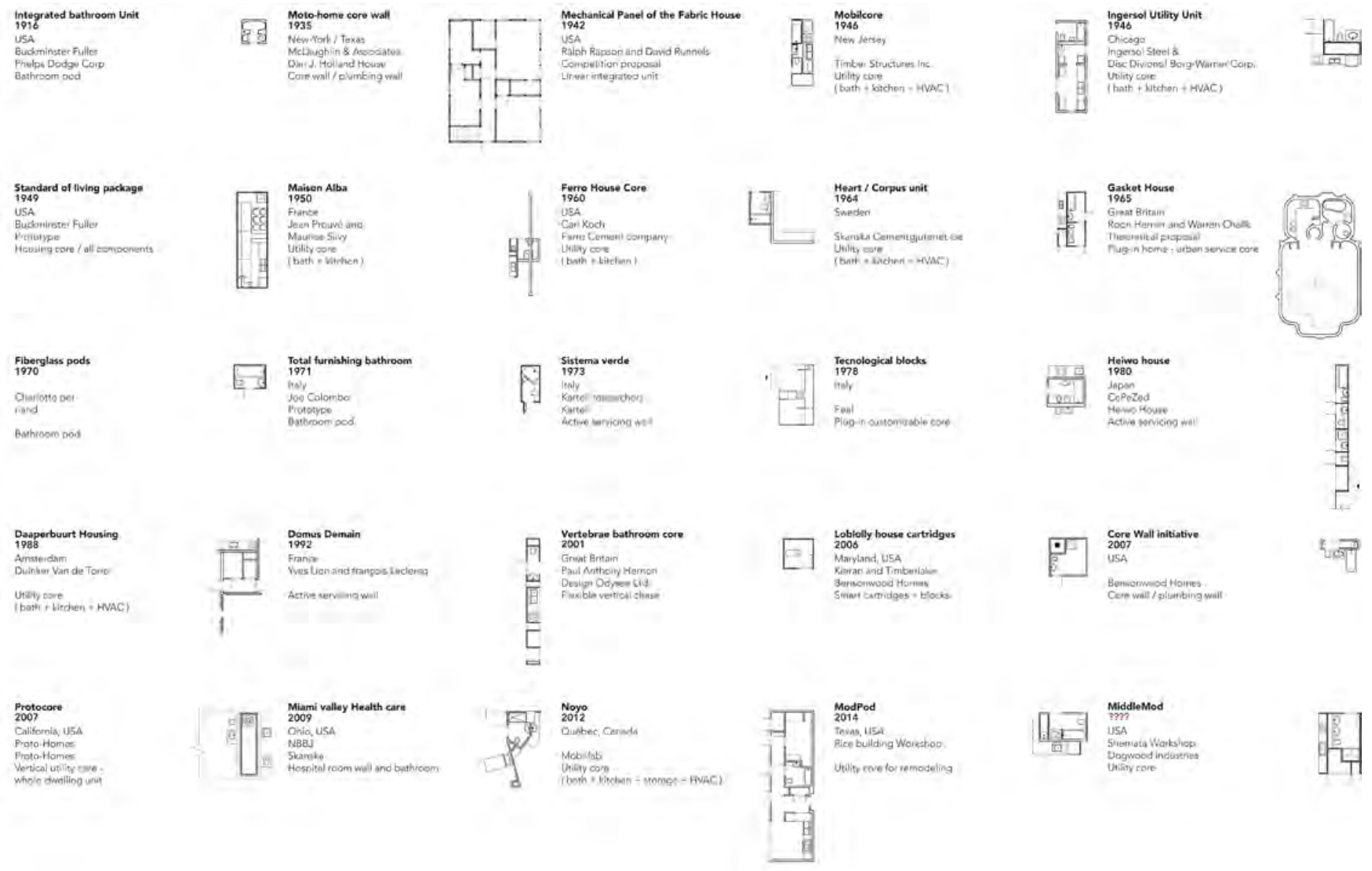

Figure 2. Timeline of selected projects. Source : (the authors)

\section{LITERATURE REVIEW}

Architectural Forum's April 1937 issue identified the long lasting analogy of the service core as the house's engine. The architectural discourse and exploration based in this argument sought to rationalize the core as a device for efficiency but also as a device for greater design flexibility and individual personalization.

Rooted in modernity the core as a device can be traced historically according to Banham's analysis of American Women's Home's central mechanical mast (Banham 1984, 97). More recently Leupen and Mooij $(2011,111)$ defined the central supply of all technical components using an even more basic analogy to the hearth. Beyond the analogical statements of engine, power plant, and hearth, a century of literature also showcases the analogies between the core's mass production and automobile production. From Fuller's folded plate bathroom ${ }^{3}$ to Kieran and Timerlake's (2004) prefabricated sub-assembly or mechanical chunk, both identified potential similarities between the core and automobiles' subassembled components, which are integrated in the factory. Besides this obvious search for construction efficiency, the major tenant of modernity as it relates to the core was zoning - the iconic central mast of Buckminster Fuller's Wichita house, idealized central zoning which became a simple rational gesture combining all services into a nucleus. The separation of servant and service spaces didactically infused architecture with rationality. Theorized and applied by Louis Kahn, the separation of two distinct spatial archetypes, while important to Kahn's specific vision, also was based on the rationalization of services (Leupen, $2006,119)$. This spatial division of two distinct functions continues to be a central theme inspired by a technical modernism. The prefabricated Quelle Bungalow developed in Germany in the early 1960s exemplifies this useful zoning as its prefabricated core establishes a customizable three-zone plan with core as both a mechanical device and a spatial separator. [fig. 1]

\footnotetext{
${ }^{3}$ Richard Buckminster fuller, "Prefabricated bathroom US 2220482" published Nov 5, 1940 - United States Patent office.
} 
Architectural Forum (1940), Kelly (1951), Diamant (1964), Dietz and Cutler (1971), Sullivan (1980), Davies (2005), and Smith (2010) all argue for a type of practicality related to streamlining all services in a packaged core. Specifically, Davies (2005) recounts the often-argued elements of timesaving, augmented quality, and compact spatial organization. From the early propositions such as the Ingersol utility unit $(1946)^{4}$ to the very recent Loblolly house by Kieran and Timberlake (2008), the integrated bathroom, kitchen, heating and mechanical supply influenced the other long-standing modernist canon of flexibility. Specific literature arguing for spatial flexibility identifies the core as a key component in allowing spatial adaptability as the core's adjacent spaces can be arranged, rearranged and modified without technical constraints. As far back as 1948, Giedion (1948, 709-711) questioned this flexibility as the core fixes the house's major components and leaves little room for flexibility over time. The question of flexibility and adaptability as it relates to service cores is a theme discussed in Jeremy Till and Tatjana Schneider's work (2005). The core allows adjacent spaces to be retrofitted, however the adaptability of the core itself is often questioned in terms of its proprietary nature.

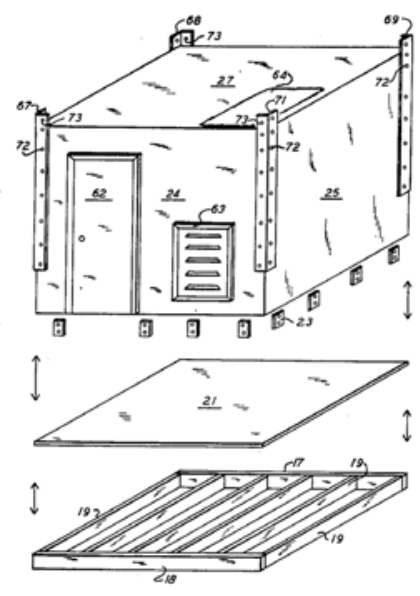

Figure 3. Building Core by Floyd E. Bigelow. Source : US patent 4470227

From Habraken's (1972) supports and infills to Teicher and Kendall's (2000) fit-outs and Till's hard and soft use (2005), all three identify some sort of service rationalization as a basis for user customization toward flexibility or adaptability. The issue of user customization most radically proposed in the typological matrices of Benros and Duarte (2009) or Noja (1978) are still not widely accepted and the core as a service pod in housing is rarely used as a design device. The literature, specifically contributions by Till and Schneider, identifies functional units, cores, units, walls and modular core units as part of larger platforms for the mass-customization of housing.

Beyond the historical narrative, recent attempts at driving prefabrication by international building authorities have again raised the potential for cores to become an important part of sensible building. Drivers such as

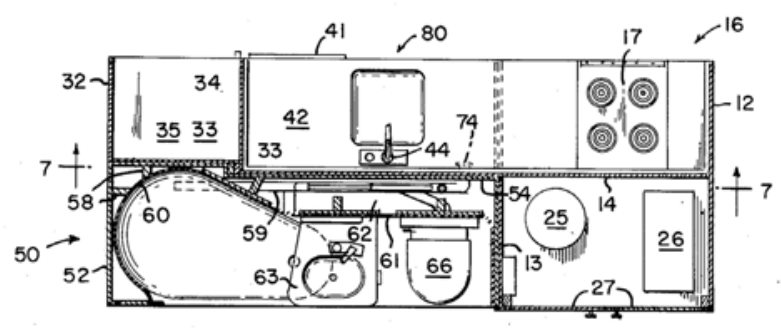

Figure 4. Prefabricated kitchen and bath unit by William E. Bain. Source: US patent 4221441

lack of qualified labor, BIM as an integrated project delivery model, and a need to enhance productivity in the building industry have brought service rationalisation back to the forefront. Professional literature has been quite proficient in this regard; articles such as Aschbrenner's (2017) "Bathroom in a Box" identify service rationalization as an innovation in construction, all the while ignoring similarities to what already has been tried. It is in the attempt to reveal what has been tested or attempted that the second half of our literature review will focus on projects - a timeline of 'core' ideas [fig. 2]. The timeline is not an exhaustive list; it is a beginning. It contributes to arguing Davies' (2005) take on the two solitudes of producer and architect and it no doubt elucidates the lack of innovation within the construction industry as a major inhibitor to the cores' development. Although the timeline of ideas does not focus specifically on inhibitors to the core's use, projects do seem to point to built-in obsolescence, lack of user input, greater upfront costs, and to the lack of systemic standardization in architecture as four potential factors hindering the service core's application. Fundamentally, though, it is Alexandra Kira's (1967) take that still seems to point out the failure of the service pod to truly infiltrate general construction "with few exceptions, the bathroom has rarely been conceived of as an entity, certainly not by the plumbing industry".

\section{The case of patents as literature review}

Complementing the literature review and posing a compelling argument for the precedent study, the investigation of patent documents revealed a number of inventions relating to the core as a either a pod, service wall, utility rooms, or mechanical boxes. The lineage from Fuller's building patent (1916) to the Building Construction Patent by $\mathrm{E}$. Gugler and J.F. Lankton (1951) and through the Building Utility Core by JC Douglas (1982), identifies the simplification of building systems into an integrated unit as a step toward simplifying construction. The overall study of patents pointed to three specific areas of ingenuity. The US4470227(1984) - Building Core patent proposed by Floyd E. Bigelow [fig. 3] addressed the issue of relating to adjacent floors and spaces. The invention proposes an open pallet type floor for simplifying shipment and system distribution as fixtures are propped up to a correct height of installation using the shipping pallet during production.

${ }^{4}$ See timeline [fig. 2] (1946) 


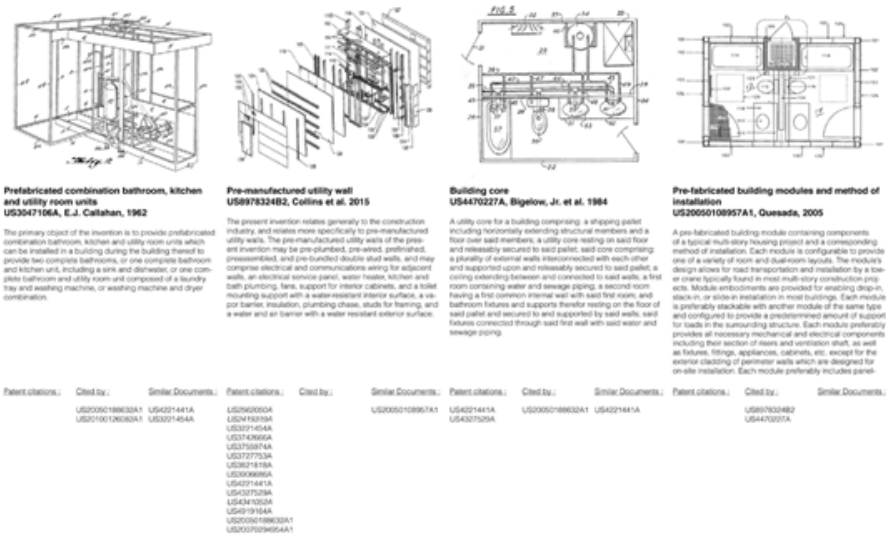

Figure 5. Excerpt from the Patent study. Source: (The authors)

A second area, which intersects with architectural discourse, is the completely integrated bath and kitchen unit. Patent US4221441 (1980) - Prefabricated Kitchen-Bath Utility System by William J. Bain, which proposes a mass-produced back-to-back kitchen and bath as the central component of a dwelling. [fig. 4]

Thirty-seven patents were identified and studied [fig. 5]. Each invention examined the problem of rationalizing services and further branched out by citing or being referenced by other inventions. The premanufactured utility wall patent US8978324B2 by Collins et al. in 2015 [fig. 6] distances itself from the ordinary pod or core by proposing a type of hub wall that relates to adjacent spaces and is customizable to any spatial configuration. The idea of a type of mechanical wall examines the potential of a more open-source core allowing users to configure and retrofit their utilities using off-the-shelf components. This proposal of a process rather than a prefabricated core seems to be an area of potential avoiding the internal obsolescence of the manufactured pod.

\section{PRECEDENT REVIEW}

Literature and patent documents offered an incomplete view of our topic as built or un-built prototypes offer a distinct area of study. Consequently, the precedent review had two objectives: the first was to complement the literature review by offering specific examples and an overall picture of the evolution of this particular segment of offsite construction, and the second was to identify possible conceptual voids to be addressed by future models. In gathering projects we focused on assembling various strategies from diverse eras and geographical contexts. As Sherwood $(1978,1)$ observed in Modern Housing Prototypes, precedent study is the basis of furthering the architectural discipline. In order to identify projects we focused on three areas: the utility core as an integrated unit of one or more services, the utility core as a device for rational as well as adaptable design, and, finally, the utility core as comprehensive serviceable unit (a manufactured technological device). We concede that this list of projects is incomplete and imagine that future research

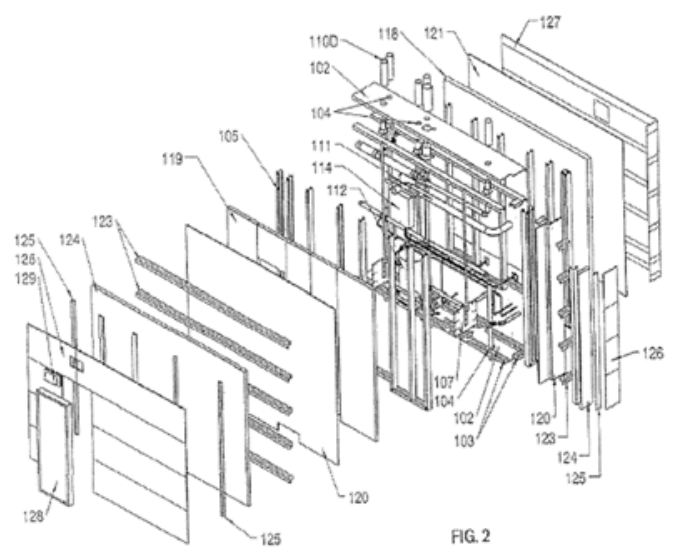

Figure 6. Pre-manufactured Utility Wall. Source: US patent 8978324B2

will continue to help us compile a more definitive catalogue. In order to extract conceptual content we focused on the issue of customization as the literature all seems to point to this as one potential inhibitor. We looked at customization on different levels. How does the core relate to overall infrastructure? What is customizable? And how does the core allow for future adaptability? The issue of a type of built-in obsolescence seems to be a major inhibitor, specifically when it pertains to manufactured cores. As technology evolves how can manufactured cores easily adapt?

Prototypes can be traced to the standardized utilities of the Fuller bathroom (1916), the Moto-home core-wall (1935), the Ingersol utility unit (1946), the Mobilcore (1946), and Prouvé's Maison Alba (1950) [fig. 2]. All predicted the combination of off-site quality with on-site flexibility. This infrastructure-to-core relationship spawned many experiments in which modular components were leveraged toward overall agility. However, service normalization, modular stacking, and utility cores have often resulted in failure to address the basic need for spatial singularity, as standardized manufacturing dimensions often dictate architectural form. The timeline in figure 2 illustrates a century of core ideas from all over the world. As for future research based on the precedent review, we continually identify projects that could be added and hope to edit a precedent analysis catalogue of utility cores.

The utility core as an object of mass production remains fairly marginally applied as architecture and construction have integrated a singular approach making each building distinct. Even as the discipline continually repeats time-consuming, wasteful and non-productive processes, architecture remains largely based on the ideal of conceptual differentiation and uniqueness (Kieran and Timberlake 2004). While this singularity is important for architects, replicability is important for factory production and improving quality. This lack of common ground has established differing strategies for the core, which were revealed in our precedent study. Architects define one strategy as the harmonization of service spaces in an attempt to create a both rational and aesthetic plan, arguing for a type of core planning. Another strategy is defined by 
producers' attempts to streamline production, material procurement and on-site coordination with maximum repetition. Another still is defined by a type of open core capable of rationalizing certain components while allowing some type of modification or adaptability.

This interesting take-away from the precedent review is what Davies (2005) identified as two competing fields (architecture and manufacturing). He argues that that while both fields were concurrent in establishing modernity, the 1950 s seem to divide the fields into one based on a type of service core rhetoric (architecture) and a second toward the simplified mass-production of bathrooms and kitchen units (manufacturing). Beyond Davies' two silos of architecture and massproduction, we have identified a third: onsite flexibility. The three narratives can be illustrated by three flagship projects.

Rapson and Runnels' Mechanical Panel of the Fabric House (1942) proposed in Architectural Forum's series on the 194(X) house is the emblem of a type of "plug-and-play" core. Fiber reinforced plastic construction is harmonized with a linear plan showcasing the core as a storage wall easily slipping into any living space defined by a modular grid establishing the typical modern dynamism [fig. 7]. From this project we can branch out into Charlotte Perriand's fiberglass cores (1970) or to metabolist megastructures or to even more lyrical projects such as Ron Herron's Gasket House (1965), where the architect controls every part of the object and, further, supplies the lifestyle description to go with it. The architectural rhetoric coined by the core is a rational use of space, the epitome of functionalist architecture; all the house's functions in one streamlined plug and play unit.

Skanska's concrete heart (1964) elucidates how the factory saw and still sees the core: simple boxes, no frills, mass produced, easy to carry and install [fig. 8]. The utility heart (Diamant 1964, 63) as an integrated box is delivered and services are plugged in on-site. This box could be mass produced and installed in collective housing blocks, maximizing assembly line production, factory profitability and construction simplicity.

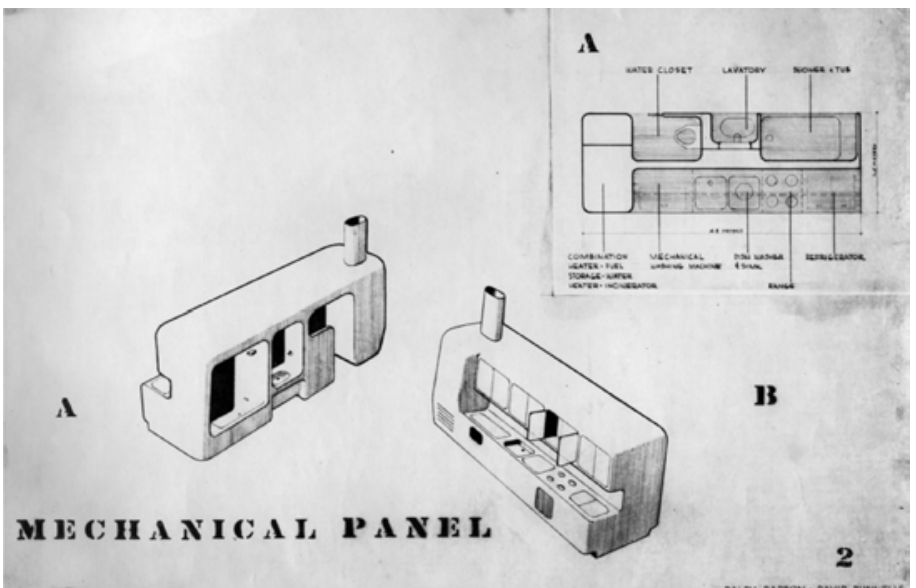

Figure 7. Mechanical Panel of the Fabric House. Source : Architectural Forum, September 1942.
MIT_n and Bensonwood Homes' corewall prototype (2007) takes an altogether new approach to the core. A wall acts as a type of motherboard hub onto which different services could theoretically be set up [fig. 9]. While this core is more about simplifying on-site construction, it allows a spatial rationalisation that has little to do with mass-production but has everything to do with a contemporary type of mass customized offsite construction as different core-walls can be modeled, produced and shipped to site to be integrated in numerous architectural typologies. This type of core can be planned to evolve over time as fixtures are added on-site as needed.

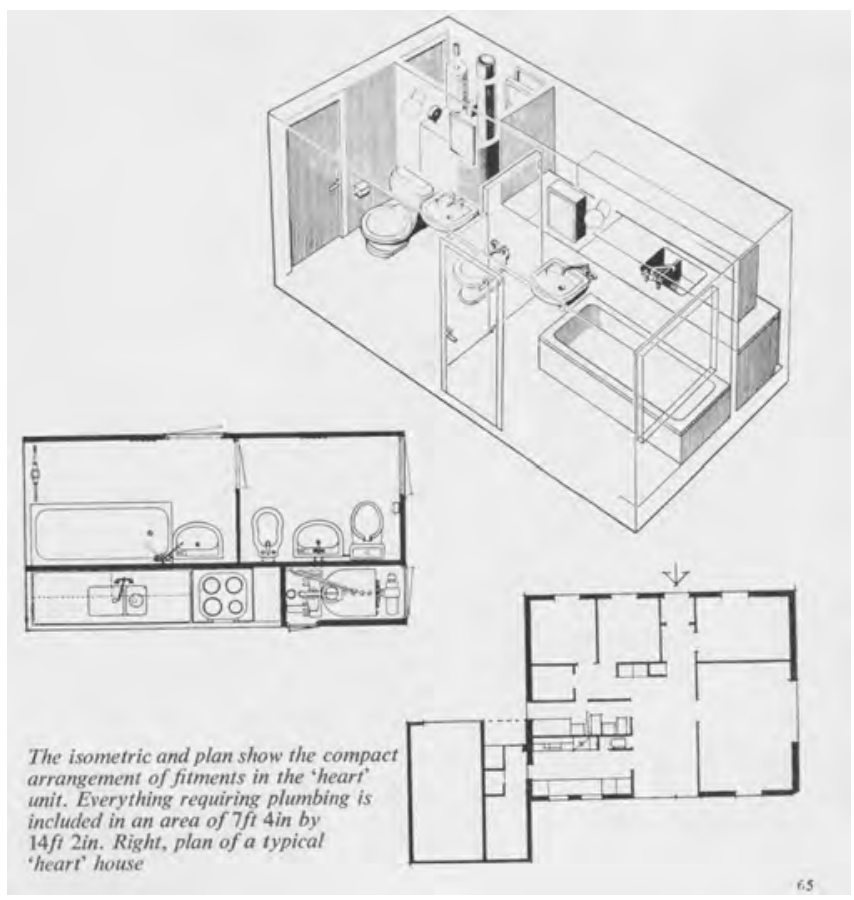

Figure 8. Skanska's Heart Unit. Source: authors' collection

The three projects showcase the inherent difficulties in adapting a service core to architecture. The architectural service core will always exist as a prototype; it is a canon of rational architectural design. As such it is neither reproducible nor an object of production. Even when it is produced off-site, it is often a one-off prototype and as such is less interesting for the future of the utility core as an object of production. In essence, either the architecture has to be repetitive, making it eaily adaptable to a mass produced component, or the core should evolve to varying lifestyles. The latter option seems to point in the direction of an informed platform capable of integrating different systems while offering a few customizable options allowing adaptability over time. The following section outlines some current explorations that seem to be continuing the long-standing definition of the core.

\section{Recent innovations}

Integration levels of the prefabricated utility components can vary in size and function, from a simple utility wall panel, a bathroom/ 


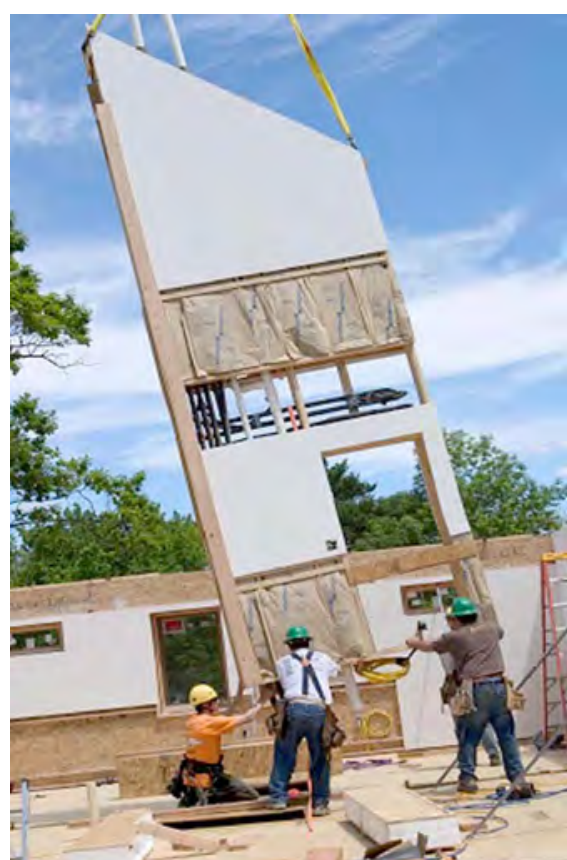

Figure 9. Core Wall. Source: Popular science, November 2006, photograph by John B. Carnett

kitchen pod, to a comprehensive service core that is comprised of a series of spatial and technical enclosures. The developments of these components is commonly considered as a time and cost saving trend due to collective characteristics of off-site construction. On one hand, the utility wall can be of a great benefit to the design and construction processes as it incorporates electrical and mechanical connections in one small area. It can increase quality, speed construction, and provide better overall housing value for the cost-conscious. On the other hand, a more elevated scale elaborates on functionality and connectivity in the form of a prefabricated bathroom/kitchen pod as a stand-alone enclosure. These pods are completed in factory then delivered to site for connection with service outlets in the house, following a plug-andplay fashion. In many cases, the utility wall can be built within the pod's structure, thus offering a more effective strategy in dealing with electrical and mechanical connections.

Following our critical appraisal of various approaches to mechanical room and utility core design, we found interesting attempts to encompass technical components within a contained prefabricated "chunk" or sub-assembly (Kieran and Timberlake, 2004, 164) that can be attached to buildings in a plug-and-play manner. This spectrum of exploration still offers a potential flexible approach that can respond at once to customization and production efficiency demands with a comprehensive approach to openly sharing a deployable core. As an example, Wikihouse on the creative commons platform showcases the potential to share building systems and could be complemented by a comprehensive take on infrastructure and its potential to adapt to changing lifestyles. ${ }^{5}$ All four projects discussed in this section employ contemporary CAD technology to collapse previous boundaries between design and production. As building modeling is becoming an integration tool, design and production fields are able to mutually inform their particular concerns making the utility core a powerful design tool

The Core Wall prototype takes on notions of flexibility on multiple levels. Given the changing nature of occupants' lifestyles, it offers potential to evolve over time. In a sense this strategy endeavours to integrate both architectural and industrial production into a manageable building component. Developed by MIT House_n and Bensonwood Homes in a collaboration between research and conventional builders, the Core Wall is designed as a key component within the Open Prototype Initiative homes. It represents a central hub around which various building systems could be designed. The structure is designed as a timber framed service panel, which includes all the house's complex components and connections, including high quality installations for heating, cooling, plumbing, ventilation, filtration, dehumidification, radon mitigation, and fire protection. Services will run to and from the core wall. The offsite constructed vertical wall is organized for a two-storey building and includes access panels to facilitate future retrofitting. The mainstream construction methods employed also avoid the excessively specific proprietary nature of certain mechanical cores that impede future adaptability [fig. 10].

As a result of their extensive exploration of technological applications in the prefabricated housing industry on various ends, from design, to fabrication, to assembly, Kieran and Timberlake designed the Loblolly House, built in an entirely atypical way. Built in 2006 on Taylors Island, Maryland, the project presented a statement towards integrated

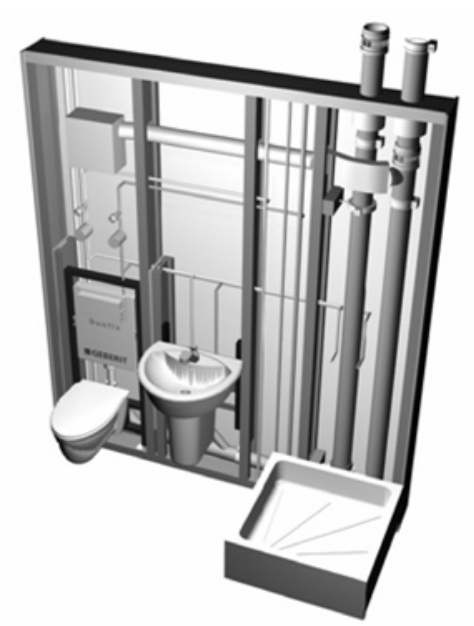

Figure 10. Digital representation of the core wall by MIT House_n and Bensonwood Homes. Source: (Open Prototype Initiative 2006)

\footnotetext{
${ }^{5}$ see technologies page of the wikihouse platform at https://wikihouse.cc/ library/technologies
} 


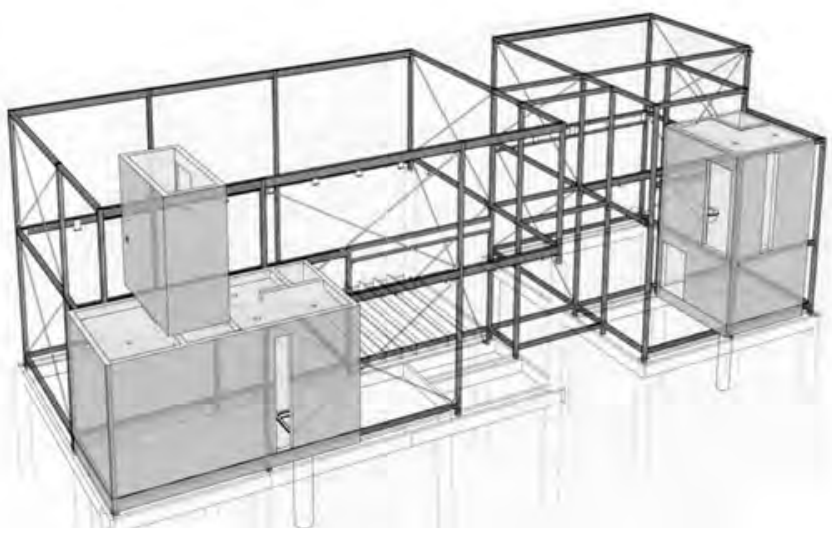

Figure 11. Representation of the 3D model of the Loblolly house, showing the skeleton, and blocks. Source: (Kieran and Timberlake 2008)

assemblies of various offsite fabricated parts. In that sense, components were articulated to four new elements of architecture: scaffold, cartridge, block, and equipment. The connections between elements were designed to facilitate assembly using only simple hand tools.

Supported by advanced BIM tools and parametric modeling strategies which required significant effort, the firm designed each of the elements digitally to insure fabrication and assembly integrity. One of the vital elements in this project is the mechanical block/module, an offsite prefabricated unit designed to contain all technical manifolds, thus organizing and managing all incoming and outgoing utility connections. In order to respond to the project's technical requirements, a series of blocks were implemented, representing a part of the comprehensive protocol developed by the architects to reach an integrated model for design, fabrication, and assembly. The mechanical blocks are timber framed modular structures that are lifted and fitted into place within the structural aluminum of the house, and connected to the rest of the house functions through smart cartridges. These cartridges coordinate mechanical components to direct the distribution of cold/hot water for faucets, radiant heating system, and enable systems integration [fig. 11].

The 3D model approach for the Loblolly house represents a comprehensive protocol for the design, integration, fabrication, and assembly of a utility core system. It combines a series of strategies that capitalize on previously described precedents with regard to architectural strategy, mass production, and design flexibility. As is the case for the Core Wall, a type of efficient, streamlined approach from 3d file to factory production is showcasing new possibilities for the utility core.

With a focus on a more inclusive approach, the ProtoCore (2007) is an original development by Proto Homes, a Los Angeles based company specializing in modern, smart, and affordable prefabricated homes. Proto Home's ${ }^{\mathrm{TM}}$ patented technology is geared toward creating homes through the application of ad bvanced digital tools in modeling and fabrication.
ProtoCore is a prefabricated centralized infrastructure silo that houses variable utilities. Designed as 3-dimensional modular box, 8-feet by 8-feet and 22-feet tall, the core serves two main purposes: technical and structural. First, the core hosts the home's mechanical, plumbing and electrical sources, such as the furnace, condensers, and water heater. Typically, toilets are all wall-mounted and are already installed in the core. All plumbing, ducts, wires and hardware are exposed and easily accessible within the core for upgrades and repairs. Second, the core acts as a major structural support, where it sits almost at the center of the foundation and then other spaces are built around it. An open plan organized around the core, the company describes living areas within the house as "hypespace". Such a design approach offers high flexibility, as well as time-based adaptability [fig. 12]. It can be argued that the ProtoCore represents an advanced approach to the plug and play architectural discourse. Branded as a vertical core, its presence is crucial for both mechanical and structural systems. It feeds all functions with necessary mechanical, electrical, and plumbing elements. Additionally, the idea of branding is essential to the ProtoCore as it complements its technical functionality with high-tech representation; an interesting selling tactic for a contemporary marketplace.

In an approach that tackles home renovation and upgrade, the ModPod (2014) is envisioned as a prefabricated residential module with a specific focus on flexibility, having the capability to fit with new, as well as existing homes. The pod contains major technical systems and finishes within a single component, thus intended to act as the heart of the home, comprising a series of spaces and functions as follows: bathroom, kitchen, office, and services [fig. 13].

Technically, the pod includes various heating, cooling, plumbing and electrical circulatory systems, in addition to providing the full kitchen and bathroom with sufficient capacity for the entire house. Components are comprised within an offsite fabricated timber frame structure, thus capitalizing on the efficiency of the process to reduce material consumption. Once delivered on site, the ModPod is easily inserted into the existing structure, weatherproofed, and then mechanical and electrical systems are coupled with existing service.

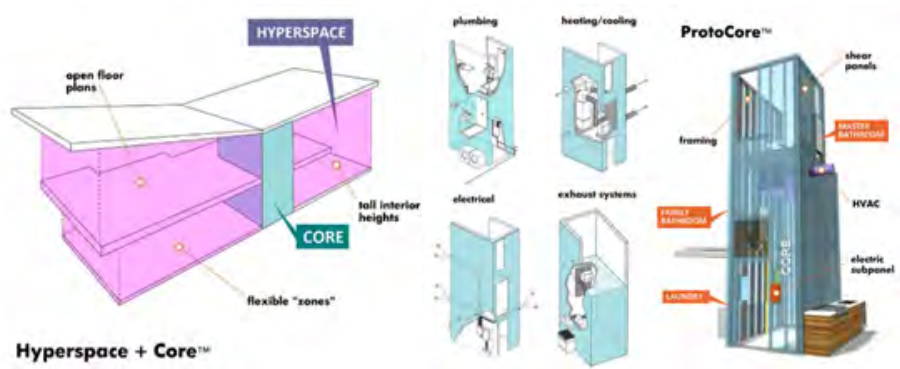

Figure 12. Drawings and diagrams of the ProtoCore by Proto Homes. Source: (Cruger 2011) 
Innovative in its use of contemporary technology to relate rational design with easy production, the ModPod clearly identifies with Skanska's Heart unit discussed previously; the modular unit functions as powerful nucleus. One of the unique features of the product is the possibility for customization, where the exterior of the ModPod could be clad in any material. Furthermore, the designers claim that it can function as a growing surface, projection wall, or backdrop for a porch.
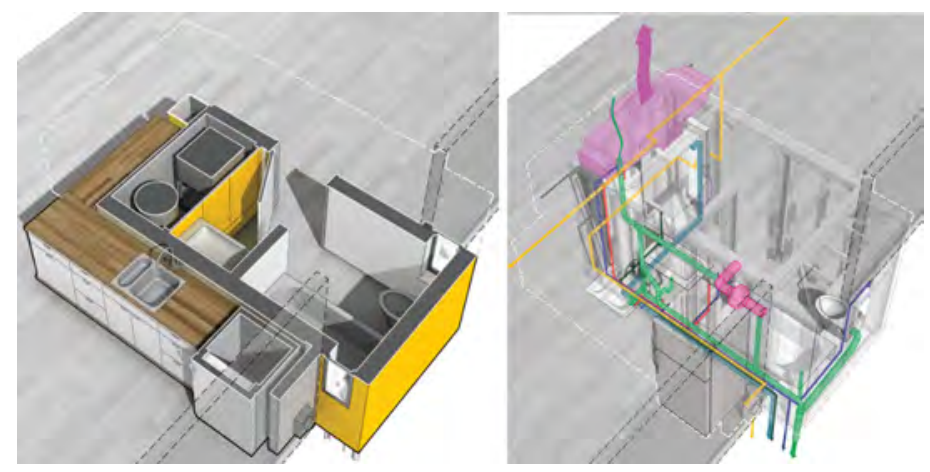

Figure 13. Representation of the ModPod showing various pod components, and a diagram of technical conduits. Source: (http:// www.modusoperations.com/2016)

\section{POTENTIALS AND CONSTRAINTS}

As noted in our literature review, there are plenty of practical reasons for producing all the major components of a house in an engine-like core. Efficiency, modularity, replicability, and ease of deployment are some of the persistent themes. Examples from the precedent review and the four previous projects in particular, Core Wall, Protocore, Loblolly House and ModPod all focus on some form of dimensional and system standardization to address efficiency while adapting to conventional building methods. This seems to have been chosen as a way to achieve widespread deployment. Even though this ideal of efficiency is well documented, the core's use as a manufactured component remains marginal: it is limited to certain types and has not percolated everyday building culture. Bathroom pods are employed regularly in repetitive typologies (hospitals, hotels), while more conceptual cores are used in architectural prototypes and one-offs looking more to smartly portray the designer's capacity to understand, control and integrate than to achieve mass-production and productivity. ${ }^{6}$

Service spaces, bathrooms, kitchens and mechanical spaces are the most contingent to technology and to retrofit. It is fairly easy to knock down a non load-bearing wall and rework adjacent spaces, whereas removing a bathroom and retrofitting a kitchen often involve major work and repositioning fixtures within the space would be difficult even with the

${ }^{6}$ see Stuck in the Middle: 8 Residences With Freestanding Service Cores Architizer at https://architizer.com/blog/freestanding-service-cores/ consulted 07-11-2017 most flexible core in the sense that wires, ducts and plumbing shafts have to be reworked. Even the most flexible core would require some major work to reposition spaces (in our study we have not found any cores that allow for this type of spatial reorganization), which is fairly common in our contemporary relationship with dwellings. The lifecycle of our commoditized environments is fairly short. At the end of the core's service life, how likely is it that the homeowner would want to put in an exact replica of the existing core? The argument can thus be made for a type of hub open to off-the-shelf components allowing owners to attain standardized services with a potential to update fixtures according to changing needs and wants.

This adaptability and evolution over time still require some type of systemic rationalization combined with a flexible strategy for retrofitting toward complete and radical changes, which is not the case for a core. The cores that we examined explore the rhetoric of plug-and-play but few examples have shown this potential in practical use. The proprietary nature of cores inhibits retrofitting, as companies rarely offer after-sale service for long periods.

The building industry is structured by long-lasting conventions of unions and subtrades which also inhibit a collective take on systems. The core was long seen as a way of streamlining this coordination issue all the while permitting some type of functional adaptability. The fixed nature of connections also limits the amount of possible retrofitting. A service core in this sense should be fitted with mechanical connectors which allow for some plug-and-play flexibility and which further could be arranged to allow for flexible arrangement with fixture placement and by a diversity of trades. In this sense, the future building core is more akin to a variable platform of interchangeable components rather than an integrated engine-like core, which one would be replace at the end of its service life.

In this case a customizable plumbing wall similar to MIT's corewall discussed previously could establish vertical and horizontal parameters of core organization allowing retrofitting to surround the core in a number of variable patterns. Clients could choose a core type according to spatial organizations and understand that retrofitting would be limited by initial choices, but they would be comforted in the fact that any retrofitting is programmed by the initial core arrangement. The core's customization could further be programmed to establish simple assembly and disassembly of components. If the core is established on a customizable platform the user could adapt and evolve the platform over time.

While the core's future as a building subassembly seems to be assured in certain segments of the building industry as the economical arguments for its production outweigh any evolution issues, their seems to be a vacant conceptual space that inhibits its use in more common construction. As one cannot prefabricate building sites, common infrastructure as it enters buildings is the first place where entanglement must be examined. Water, electrical and sewage systems come into the building at various points and dictate a disparate distribution. The core could be envisaged first as an infrastructure connector, a hub that unites different services into one intelligent distribution of coordinated parts. 


\section{DISCUSSION AND CONCLUSION: PAST, PRESENT AND FUTURE}

Our literature and precedent review illustrated the rich and diverse number of strategies to simplify service distribution in buildings. The central engine-like utility core exemplifies the type of efficient rational thought that percolated building culture from the automobile's mass production. This type of core-to-space relationship is still seen today as a way of progressing building culture. However, the mass produced core remains an illustration of divergent fields of action: the rhetorical core in architecture which is rarely mass produced but exemplifies the architect's position for rational thought, while the mass produced industrial core requires an identifiable reproduced building type to function. Further, both strategies, the architectural and the industrial, never achieve streamlined building and rarely offer the promised flexibility. Even in the case of hotel retrofitting, the installed core would probably have to be dumped at the end of its service life. Is it possible then to imagine a core that integrates its end of service life into an equally rational mode of adaptability?

As digital design and fabrication tools have democratized a model of shared methodologies for construction, building cores could also be shared among users thus helping initiate core cross pollination and integration. Within a building system, the open-source building core could be conceived as a digitally fabricated core wall, which allows for pipes to be placed, removed and replaced without rebuilding the wall.

For too long the core has reproduced traditional building methods in a factory. We believe it is perhaps time for today's technology to dictate a new type of service core capable of redefining the role of utilities with the building in a more intelligent and specifically intelligible fashion. 


\section{REFERENCES}

Aschbrenner, Joel. "Bathroom in a Box : Contractors Snatching them Up" DesMoines Register. May 5, 2017 retrieved on 2 June, 2017 from http://www.desmoinesregister.com/story/money/business/ biz-buzz/2017/05/05/bathroom-box-contractors-increasingly-turningprefabrication/309901001/

Benros, D. and J.P. Duarte "An integrated system for providing mass customized housing." Automation Construction. No.18. 2009. 210-230.

Buckminster, Richard Fuller. "Prefabricated bathroom US 2220482" published Nov 5, 1940 - United States Patent office.

Cruger, Roberta. "Https://www.treehugger.com/sustainable-productdesign/proto-homes-flexible-spaces-and-efficient-core.html." Treehugger. March 19, 2011. Accessed June 20, 2017. https://www. treehugger.com/sustainable-product-design/proto-homes-flexiblespaces-and-efficient-core.html.

Davies, Colin. The Prefabricated Home. Reaktion books. 2005.

Dawn, Stover. "Building Blocks - The House of the Future." Popular Science. November, 2006.

Diamant, R.M.E. Industrialised Building, volumes 1,2,3. Ilife Books Ltd. 1964.

Dietz, Albert G.H. and Laurence S. Cutler. Industrialized Building Systems for Housing. MIT Press. 1971.

Ebert, Carola. "Into the great wide open: The West-German modernist bungalow of the 1960s as a psycho-political re-creation of home." Muli, Volume 2, Number 1, Winter 2008, 35.

Giedion, Sigfried. Mechanization Takes Command. W-W Norton \& Company. 1948.

Habraken, N. John. Supports: an Alternative to Mass Housing. The Architectural Press. Praeger. 1972.

"The Integrated House: 'power plant'” Architectural Forum. April,1937.

Kelly, Burnham. The Prefabrication of Houses. MIT Press and John Wiley and Sons. 1951.

Kendall, Stephen and Jonathan Teicher. Residential Open Building. Spon Press, 2000.

Kieran, Stephen and James Timberlake. Loblolly House Elements of a New Architecture.Princeton Architectural Press. 2008.

Kieran, Stephen and James Timberlake. Refabricating Architecture: How Manufacturing Methodologies Are Poised to Transform Building Construction. McGraw-Hill. 2004.

Kira, Alexandra. The Bathroom. Viking Press. 1967.
Leupen, Bernard and Harald Mooij. Housing Design - A Manual. NAi Publishers. 2011

Lupton, Ellen and J. Abbott Miller. The Bathroom and The Kitchen and The Aesthetics of Waste, MIT Visual Arts Centre, Princeton Architectural Press. 1992. 35.

Modpod. January 2014. Accessed June 20, 2017. http://www. modusoperations.com/ 2016.

Noja, C., "Prefab Abitazioni in Lomarbardia" (Low-Cost Housing Standard Projects), in PREFAB Special edition of Domus magazine of Architecture, Design and Art, No. 587. October, 1978.

Open Prototype Initiative: Transforming the Way America Builds Homes. PDF. www.openprototype.com.

Pan, W., A.R.J. Dainty, and A.G.F. Gibb, "Encouraging appropriate use of Osite Production (OSP): perspectives of designers." 2nd CIB Student Chapter International Symposium, 30-31 Oct 2004, Beijing, China, pp.125-36.

Schneider, Tatjana and Jeremy Till, "Flexible housing; the means to the end." Architectural Research Quarterly, vol. 9 no3/4, 2005, 287-296.

Schneider, Tatjana and Jeremy Till, "Flexible housing; opportunities and limits", Architectural Research Quarterly, vol. 9 no3/4, 2005, 157-166.

Sherwood, Roger. Modern Housing Prototypes. Harvard University Press, 1978.

SmartMarket Report, Prefabrication and Modularization: Increasing Productivity in the Construction Industry, McGraw-Hill Construction retrieved on June 25, 2017 from - http://www.modular.org/htmlPage. aspx?name=McGrawHill_Prefabrication .

Smith, Ryan. Prefab Architecture. John Wiley and Sons. 2010.

Sullivan, Barry James. Industrialization in The Building Industry. Van Nostrand Reinhold Company. 1980.

Topping R., Lawrence T., Spencer J., Larson K., McLeish T.J. Organizing Residential Utilities : A New Approach to Housing Quality, US Department of Housing and Urban Development, 2004. Retrieved on June 25, 2017, https://www.huduser.gov/portal/Publications/pdf/ DisentanglingUtilities.pdf. 